\title{
Parameter Identification of a Cardiovascular Model for the Estimation of Ventricular Pressure on Aortic Stenosis
}

\author{
Kimi P. Owashi ${ }^{1}$, Arnaud Hubert ${ }^{1}$, Elena Galli ${ }^{1}$, Erwan Donal $^{1}$, Alfredo I. Hernández ${ }^{1}$, Virginie Le \\ Rolle ${ }^{1}$ \\ ${ }^{1}$ Univ Rennes, CHU Rennes, Inserm, LTSI - UMR 1099, F-35000 Rennes, France
}

\begin{abstract}
This paper proposes a model-based estimation of left ventricular $(L V)$ pressure and pressure-strain loops (PSL), adapted to patients with aortic stenosis $(A S)$. We propose a model of the cardiovascular system composed of $i$ ) cardiac electrical activity, ii) elastance-based mechanical activity, iii) systemic and pulmonary circulations and iv) heart valves. An identification strategy was implemented using a leave-one-out cross validation approach in order to estimate patient-specific LV pressures for 4 patients suffering from AS. LV pressure curve is then used for the estimation of PSL. A close match was observed between experimental and simulated LV pressures for all 4 patients. The global root mean square error (RMSE) is equal to 20.34 ( \pm 5.34$)$ mmHg. RMSE for systolic and diastolic arterial pressures are respectively $0.89( \pm 0.79)$ and $0.2370( \pm 0.18) \mathrm{mmHg}$. Mean PSL areas calculated for experimental and modelbased pressures are respectively $1965.6 \pm 206.77 \mathrm{~cm}^{2}$ and $2206.4 \pm 289.64 \mathrm{~cm}^{2}$. The proposed model-based method may be a useful tool to improve the noninvasive estimation of $L V$ pressure and the characterization of $L V$ function on patients with severe AS.
\end{abstract}

\section{Introduction}

Aortic stenosis (AS) is one of the most common cardiac valve diseases and is characterized by a restriction of blood flow from the left ventricle to the aorta, due to a narrowing of the valve opening. The development of AS is usually accompanied by systolic and diastolic dysfunction [1] and novel non-invasive tools are required to improve the evaluation of cardiac function in AS patients. Quantitative evaluation of left ventricular (LV) function is still challenging, particularly from echocardiography, because of the lack of consideration of load conditions [2]. The area of left ventricular (LV) pressure/strain loop (PSL) has been recently proposed as a surrogate estimation of the power over the cardiac cycle [3]. Although PSL analysis appears as a promising approach $[4,5]$, it requires the observation or estimation of an LV pressure signal. However, the observation of LV pressure is difficult to perform clinically because it requires an invasive, intraventricular measurement. Although methods for the estimation of an LV pressure curve from noninvasive measurements have been proposed [3], their accuracy has not been verified in the presence of cardiac valve pathologies.

The objective of this paper is to propose a noninvasive approach to estimate robust LV pressure curves in the case of AS. A cardiovascular system model and a parameter identification method are proposed in this sense. An integrated model of the cardiovascular system (CVS) is proposed, based on the coupling of several sub-models: $i$ ) cardiac electrical system [6,7], $i i$ ) elastance-based cardiac cavities [8-10], iii) systemic and pulmonary circulations [11], and $i v$ ) heart valves [12]. An identification strategy was implemented using a leave-one-out cross validation approach in order to provide patient-specific modelbased LV pressure of 4 AS patients, from aortic valve area, systolic and diastolic pressures. Finally, pressure/strain loops were evaluated from the model-based estimation of LV pressure and compared to PSL assessed from invasive experimental pressures.

\section{Methods}

\subsection{Dataset}

The dataset used in this study contains 4 patients suffering severe AS (aortic valve area $\leq 1 \mathrm{~cm}^{2}$ ). The study protocol was approved by the Rennes University Hospital ethics committee (authorization number: 2014-A01331456). The experimental invasive dataset includes the ECG, the measured ventricular $P_{L V}^{e x p}$, systolic $P_{a o, s y s}^{e x p}$ and diastolic $P_{a o, d i a s}^{\exp }$ arterial pressures. For this purpose, left heart catheterization (LHC) was performed in all the patients via a retrograde access from the radial artery with a 5 French Judkin R4 catheter (ICU Medical, San Clemente, CA, USA) placed at the mid LV cavity using fluoroscopic screening. All patients underwent a standard TransThoracic Echocardiography (TTE) using a Vivid S6, E7 


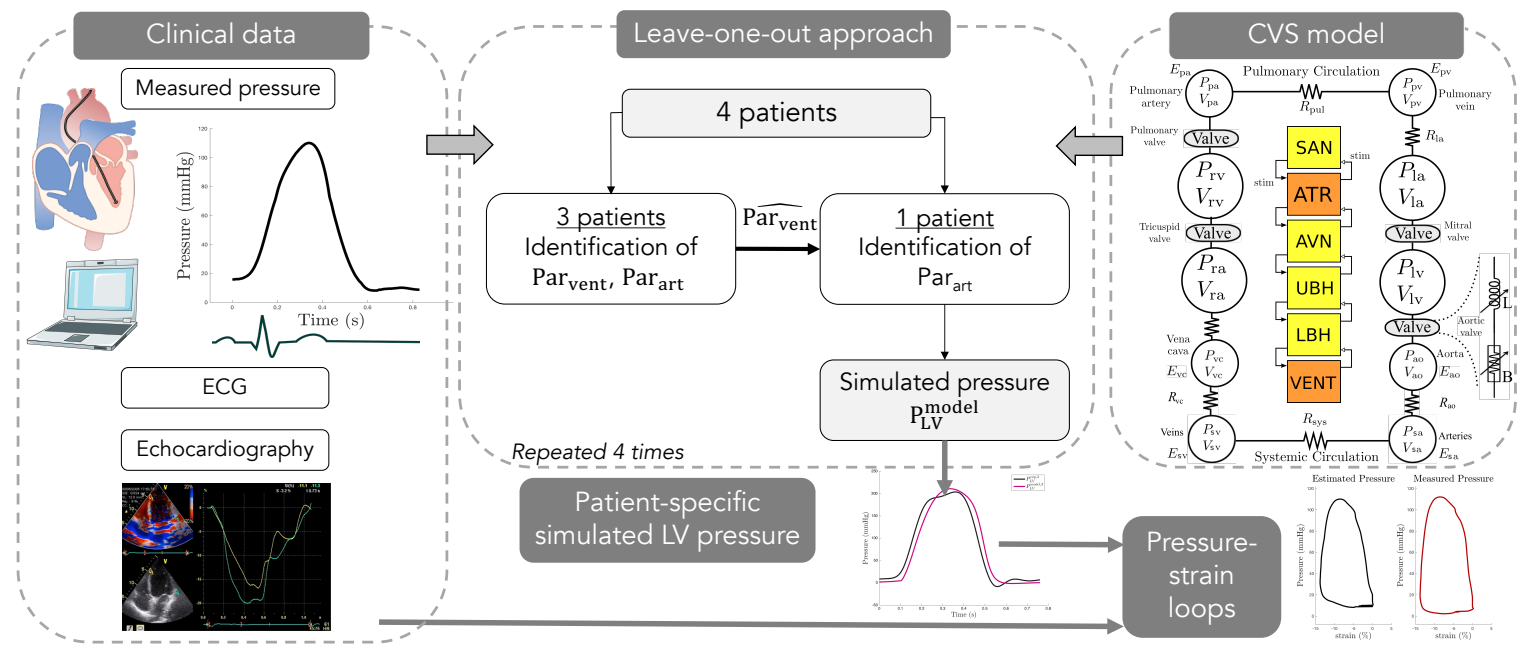

Figure 1. Methodology description. Dataset acquisition (left). Parameter identification strategy using a leave-one-out cross validation approach (middle). Closed-loop model of the cardiovascular system (right). Sinoatrial node (SAN), atria (ATR), atrioventricular node (AVN), upper bundle of His $(\mathrm{UBH})$, bundle branches $(\mathrm{LBH})$, and ventricles (VENT).

or E9 ultrasound system (General Electric Healthcare, Horten, Norway), in order to extract regional myocardial strain curves and estimate the aortic valve area AVA $\left(\mathrm{cm}^{2}\right)$.

\subsection{Model description:}

Cardiac electrical system: A set of interconnected cellular automata, adapted from [6,7], represents the cardiac electrical activity of the model. Each automaton represents different cardiac regions that cycle between four electrical activation states: slow diastolic depolarization (SDD), upstroke depolarization period (UDP), absolute refractory period (ARP) and relative refractory period (RRP).

Elastance-based cardiac cavities: The CVS model includes time-variant elastance cardiac cavities, controlled by driving functions. The "double Hill" $e_{l v}$ [13] and the Gaussian driving functions $e_{l a}$ were selected to represent the contraction and relaxation of ventricles and atria respectively. Ventricle pressure $(P)$ is calculated using a linear relationship of its volume $(V)$ and its elastance $(E)$ associated with systole $(e s)$ and a non-linear pressurevolume relationship associated with diastole $(e d)$ [14]:

$$
\begin{gathered}
P_{e s, l v}(V, t)=E_{e s, l v}\left(V(t)-V_{d, l}\right) \\
P_{e d, l v}(V, t)=P_{0, l v}\left(e^{\lambda_{l v}\left(V(t)-V_{0, l}\right)}-1\right) \\
P_{L V}^{\text {model }}(V, t)=e_{l v}(t) P_{e s, l v}(V, t)+\left(1-e_{l v}(t)\right) P_{e d, l v}(V, t)
\end{gathered}
$$

Systemic and pulmonary circulations: Concerning the circulatory models [11], pressures on the cardiac or vessel chambers are calculated as a linear pressure-volume relationship, similar to Eq. 1. The pressure gradient across chambers and the corresponding vascular resistance are then used to calculate flows as: $Q=\Delta P / R$. Volumes of each cardiac and vessel chamber is computed from the integral of their respective net flow. The circulatory model allows for the simulation of systolic and diastolic aortic pressures $\left(P_{a o, s y s}^{\text {model }}\right.$ and $\left.P_{a o, d i a s}^{\text {model }}\right)$.

Cardiac valves: Based on [12], a detailed model describing the valve dynamics $\xi(t)$ was coupled to our CVS model to represent the heart valves motions:

$$
\frac{d \xi}{d t}=\left\{\begin{aligned}
(1-\xi) K_{v o} \Delta P & \text { if } \Delta P>0 \\
\xi K_{v c} \Delta P & \text { if } \Delta P \leq 0
\end{aligned}\right.
$$

In response to the pressure gradient $\Delta P$ across the valve, $K_{v o}$ and $K_{v c}$ are the rate coefficients for valve opening and closure, respectively. $\Delta P$ is calculated by the Bernoulli equation. The effective cross-sectional area of the valve $A_{\text {eff }}$ is related with the effective length of the valve $l_{\text {eff }}$ and $\xi(t)$ :

$$
A_{e f f}(t)=\left[A_{e f f, \max }(t)-A_{e f f, \min }(t)\right] \xi(t)+A_{\text {eff,min }}(t)
$$

\subsection{Parameter identification}

The objective of this section is to propose a method that could be used to provide a model-based estimation of LV pressure from systolic and diastolic pressures. A leaveone-out cross validation approach was used to perform LV pressure estimation. For all patients, the maximum effective area $A_{\text {eff,max }}^{l}$ parameter was fixed to the observed 
AVA, measured from TTE. Available data from the 4 patients were divided randomly into two sets: training (3 patients) and test ( 1 patient). In the training set, patientspecific parameters of ventricular $\left(\operatorname{Par}_{L V}\right)$ and circulatory $\left(\right.$ Par $\left._{\text {art }}\right)$ models are identified for each patient in order to minimize the error function:

$$
\epsilon_{\text {training }}=\epsilon_{L V}+\epsilon_{\text {sys }}+\epsilon_{\text {dias }}
$$

with

$$
\begin{aligned}
\epsilon_{\text {sys }} & =\left|P_{a o, \text { sys }}^{\text {exp }}-P_{\text {ao,sys }}^{\text {model }}\right| \\
\epsilon_{\text {dias }} & =\left|P_{\text {ao,dias }}^{\text {exp }}-P_{\text {ao,dias }}^{\text {model }}\right| \\
\epsilon_{L V} & =\frac{1}{T_{c}} \sum_{t_{e}=0}^{T_{c}-1}\left|P_{L V}^{\text {exp }}\left(t_{e}\right)-P_{L V}^{\text {model }}\left(t_{e}\right)\right|
\end{aligned}
$$

where $t_{e}$ corresponds to the time elapsed since the onset of the identification period and $T_{c}$ is the duration of a cardiac cycle. This error function was minimized using evolutionary algorithms (EA) [15] . Then, the ventricular parameters of the test patient were fixed to the average values over the 3 other patients $\widehat{\operatorname{Par}_{L V}}=\operatorname{mean}\left(\operatorname{Par}_{L V}\right)$ and circulatory parameters Par $_{\text {art }}$ were identified by minimising the error function:

$$
\epsilon_{\text {test }}=\epsilon_{\text {sys }}+\epsilon_{\text {dias }}
$$

The whole process was repeated four times and LV pressure $P_{L V}^{\text {model }}$ was simulated using the best set of parameters found for each patient.

\subsection{Pressure-strain loops}

As performed in previous work of our team [16], pressure/strain loops were determined from the mean myocardial strain, calculated from all segmental curves, using both measured and model-based pressures. The area of each loop was calculated using the rectangle method, considering small increments of ventricular pressure in order to insure a precise determination. The same method was used for the calculation of the loop area for measured and model-based pressures.

\section{Results}

\subsection{Comparison with experimental data}

From the identification process we obtained a patientspecific simulated LV pressure signal. Figure 2 compares the simulated and experimental LV pressure of the $4 \mathrm{AS}$ patients. A close match was observed between LV experimental and simulated pressures since, for all patients, root mean square error (RMSE) is equal to $20.34( \pm 5.34)$
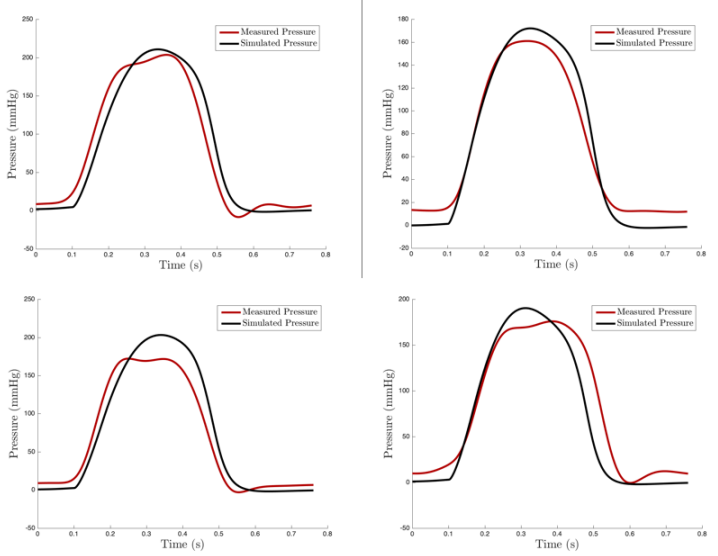

Figure 2. LV pressure of 4 patients: i) simulated curve (black), ii) experimental curve (red).

mmHg. The mean RMSE for systolic and diastolic arterial pressures are respectively $0.89( \pm 0.79)$ and $0.2370( \pm$ $0.18) \mathrm{mmHg}$.

Figure 3 illustrates the simulated and experimental PSL of all the patients. Similar morphologies between experimental and simulated PSL can be noticed in each patient as a consequence of the close match obtained between invasive experimental and patient-specific simulated LV pressures. The mean PSL areas calculated for experimental and model-based pressures are respectively $1965.6 \pm 206.77$ $\mathrm{cm}^{2}$ and $2206.4 \pm 289.64 \mathrm{~cm}^{2}$.
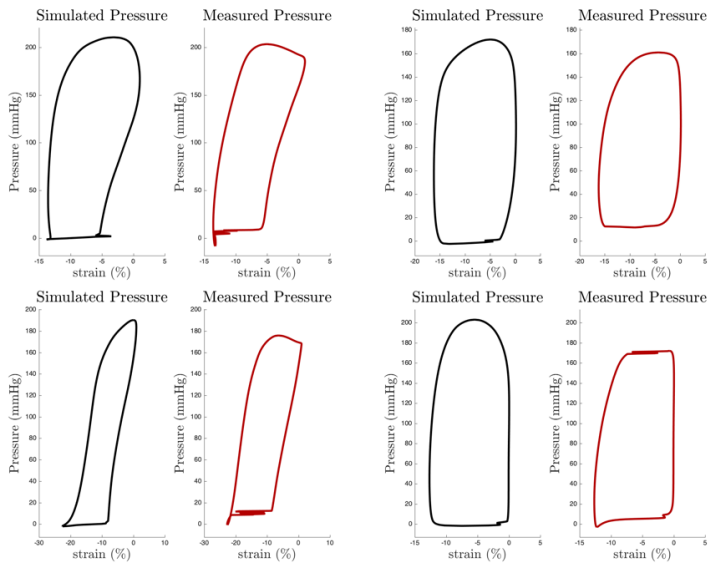

Figure 3. Pressure-strain loop of the 4 patients: i) simulated curve (black), $i$ ) experimental curve (red).

\section{Discussion}

The main contribution of this work concerns the proposal of the parameter identification procedure, applied to an integrated CVS model, able to reproduce LV pressure specifically to each AS patient, by non-invasive pro- 
cedures.

The coupling of the CVS model, developed by our group [6-11], to heart valve model, proposed by [12], allows to correctly represent the influence of valve motion on hemodynamic parameters, obtaining the expected results in the AS case. Results from the proposed leave-one-out crossvalidation indicate that the model, only evaluating Par Prt $_{\text {art }}$ from a given patient, is able to provide a non-invasive estimation of the LV pressure waveform that agrees with the experimental curve. For this purpose, only 3 non-invasive data, that can be easily acquired from any patient, are necessary: the AVA and the systolic $\left(P_{a o, s y s}^{e x p}\right)$ and diastolic $\left(P_{\text {ao,dias }}^{e x p}\right)$ arterial pressures.

This paper presents a method for the estimation of LV pressure and PSL, based on a physiological model with promising clinical applications. PSL area has also been used to calculate cardiac work indices in several pathologies providing, for example, a new opportunity to better predict the response to cardiac resynchronization therapy [16]. Because of the consistency between measured and simulated LV pressures, model-based PSL area markers appear as a robust surrogate estimation and as promising tools to provide prognostic information in AS patients.

One of the main limitations of this work is the limited number of patients included. Further work is necessary in order to apply the training phase on a higher number of patients, in particular to learn a set of robust $\operatorname{Par}_{L V}$ parameters.

\section{Conclusion}

Assessment of LV pressure and PSL loops, for patients with severe AS, can be improved by using patient-specific models of the cardiovascular system. Future works will be dedicated to more extensive evaluations including a greater population of patients.

\section{Acknowledgments}

This work was supported by the French National Research Agency (ANR) (ANR-16-CE19-0008-01) (project MAESTRo) and the French Brittany council (ADvICE project).

\section{References}

[1] Rajappan, et al. Mechanisms of coronary microcirculatory dysfunction in patients with aortic stenosis and angiographically normal coronary arteries. Circulation 2002;105:470476.

[2] Galli E, Leclercq C, Donal E. Mechanical dyssynchrony in heart failure: Still a valid concept for optimizing treatment? Archives of Cardiovascular Diseases January 2017; 110(1):60-68.

[3] Russell K, et al. Assessment of wasted myocardial work: a novel method to quantify energy loss due to uncoordinated left ventricular contractions. Am J Physiol Heart Circ Physiol 2013;305:H996?H1003,.

[4] Russell K, et al. Mechanism of prolonged electromechanical delay in late activated myocardium during left bundle branch block. American Journal of Physiology Heart and Circulatory Physiology December 2011; 301(6):H2334-H2343.

[5] Vecera J, et al. Wasted septal work in left ventricular dyssynchrony: a novel principle to predict response to cardiac resynchronization therapy. European Heart Journal Cardiovascular Imaging February 2016;17(6):624-632.

[6] Hernández AI, et al. Model-based interpretation of cardiac beats by evolutionary algorithms: signal and model interaction. Artificial Intelligence in Medicine 2002;26(3):211235.

[7] Le Rolle V, et al. Model-based analysis of myocardial strain data acquired by tissue doppler imaging. Artificial Intelligence in Medicine 2008;44:201-19.

[8] Romero-Ugalde HM, et al. Model-based design and experimental validation of control modules for neuromodulation devices. Biomedical Engineering IEEE Transactions on 2015;63(7):1551-1558.

[9] Calvo M, et al. Model-based analysis of the autonomic response to head-up tilt testing in Brugada syndrome. Comput Biol Med 2018;103:82-92.

[10] Calvo M, et al. Recursive model identification for the analysis of the autonomic response to exercise testing in Brugada syndrome. Artif Intell Med 2018;.

[11] Ojeda D, et al. Sensitivity analysis and parameter estimation of a coronary circulation model for triple-vessel disease. IEEE Transactions on Biomedical Engineering 2014; 61(4):1208-1219.

[12] Mynard JP, Davidson MR, Penny DJ, Smolich JJ. A simple, versatile valve model for use in lumped parameter and one-dimensional cardiovascular models. Int J Numer Meth Biomed Engng 2012;;28:626?641.

[13] Stergiopulos N, et al. Determinants of stroke volume and systolic and diastolic aortic pressure. Am J Physiol 1996;; 270:H2050-H2059.

[14] Chung D, et al. A dynamic model of ventricular interaction and pericardial influence. American Journal of Physiology Heart and Circulatory Physiology 1997;272(6):H2942H2962.

[15] Goldberger AL, et al. What is physiologic complexity and how does it change with aging and disease? Neurobiology of aging 2002;23(1):23-26.

[16] Hubert A, Le Rolle V, Leclercq C, Galli E, Samset E, Casset C, Mabo P, Hernandez A, Donal E. Estimation of myocardial work from pressure-strain loops analysis: an experimental evaluation. Eur Heart J Cardiovasc Imaging 2018;

Address for correspondence:

Kimi Owashi

LTSI. Université de Rennes 1, campus de Beaulieu, Bâtiment 22.

35043 Rennes, France.

kimi-piedad.owashi@univ-rennes1.fr 